sexual behaviour. Our objective was to assess whether contextual exposure to social disorder is correlated with self-reported sexual intercourse among adolescent girls.

Design and Methods Girls $(\mathrm{N}=48)$, aged $14-16$, were recruited from a single geographic urban area and monitored for 1 week using a GPS-enabled cell phone. Adolescents completed an ACASI survey on self-reported sexual intercourse in the last 30 days. In addition to recorded home and school address, phones transmitted location data every 5 min (travel points). Using ArcGIS, we defined social disorder as aggregated point-level Unified Crime Report data within a 200 metre Euclidian buffer from home and each travel point. Using Stata, we analysed how social disorder exposures differed among girls who reported having sex or not.

Results Girls reporting sexual activity spent less time at home $30.9 \%$ vs $55.7 \%, \mathrm{p}<0.001)$ and more time at least $5 \mathrm{~km}$ away from home $(29.5 \%$ vs $12.4 \%, p=0.01)$. Girls who reported sex in the last 30 days lived in areas of higher social disorder than girls not reporting sex $(p=0.01)$. There were no significant differences in exposure to social disorder based on travel data among girls reporting sex or not, however. Exposure to social disorder varied by school/non-school day as well as time of day. [Abstract O1-S11.05 figure 1].

Conclusions Even within neighbourhoods, social disorder surrounding the home as defined by crime within a 1-block radius correlates with adolescent sexual intercourse behaviour. Although adolescents reporting having sex are less likely to spend time at home and more likely to be further from home than girls not reporting sex, the areas where they travel to are not different in terms of social disorder.

\section{1-S11.06 BURDEN OF GENITAL DISCHARGE PATHOGENS AND ASSOCIATED CHARACTERISTICS OF ASYMPTOMATIC HIV-INFECTED PATIENTS IN JOHANNESBURG, SOUTH AFRICA}

doi:10.1136/sextrans-2011-050109.66

${ }^{1} \mathrm{D}$ Lewis, ${ }^{2} \mathrm{~T}$ Chirwa, ${ }^{1} \mathrm{~V}$ Msimang, ${ }^{1} \mathrm{~F}$ Radebe, ${ }^{3} \mathrm{M}$ Kamb, ${ }^{4} \mathrm{I}$ Sanne, ${ }^{4} \mathrm{C}$ Firnhaber. ${ }^{1}$ National Institute for Communicable Diseases, National Health Laboratory Service, Sandringham, South Africa; ${ }^{2}$ University of the Witwatersrand Johannesburg, South Africa; ${ }^{3}$ Centers for Disease Control and Prevention, Atlanta, USA; ${ }^{4}$ Right to Care, Johannesburg, South Africa

Background The prevalence of asymptomatic STIs and urethritis/ cervicitis pathogen-associated patient characteristics were determined among patients attending a HIV treatment centre in Johannesburg.

Methods Consenting consecutive HIV-infected patients, asymptomatic for symptoms/signs of genital discharge, were screened over 12 months for gonorrhoea, trichomoniasis, chlamydial and Mycoplasma genitalium infections using a real-time PCR assay. Bacterial vaginosis (BV) and Candida were detected by microscopy (women only). Serological assays diagnosed syphilis (RPR/TPPA) and herpes simplex type 2 (IgG ELISA) infections. Patients returned at 2 weeks; those with positive results were treated and given contact slips for partners. If available, patients' most recent CD4 (83\%) and viral load (VL) $(60 \%)$ results were recorded. Demographic, clinical and behavioural data were collected by nurse-administered questionnaire. A descriptive analysis was conducted to obtain frequency distributions of patient and STI prevalence data. Associations were investigated using the $\chi^{2}$ test at a $5 \%$ level of significance. A multiple logistic regression model was fitted to find factors associated with urethritis/cervicitis pathogens.

Results 1109 patients were enrolled (551 men, 558 women). Compared with men, women were younger with a mean age [SD] of 35.0 [7.3] vs 37.9 [7.9] years $(p<0.001)$, reported more STIs in the past year $(65.5 \%$ vs $56.5 \%$, $\mathrm{p}=0.002)$, were less likely to know their partner's HIV status (53.1\% vs $62.3 \%$, $\mathrm{p}=0.007)$, were more likely to be on HAART $(70.4 \%$ vs $59.7 \%, \mathrm{p}<0.001)$ with an undetectable VL (81.0\% vs $69.9 \%, p<0.001)$ and a higher mean [SD] CD4 count of 346 [203] vs 232 [173] cells/mm3 $(\mathrm{p}<0.001)$. Urethritis/cervicitis pathogens were detected in 119/558 (21.3\%) women and 90/550 $(16.4 \%)$ men $(p=0.035)$. BV and Candida were detected in 155 $(28.0 \%)$ and 101 (18.3\%) women, respectively. Detection of urethritis/cervicitis pathogens was associated with recent sexual intercourse with a regular partner (adjusted OR, aOR 1.64, 95\% CI $1.08 \%$ to $2.48 \%$ ). Trichomoniasis was associated with female gender (aOR 2.45, 95\% CI $1.39 \%$ to $4.32 \%$ ) and sub-optimal condom use with regular partners (aOR 2.04, 95\% CI $1.23 \%$ to $3.41 \%$ ).

Conclusions Urethritis/cervicitis pathogens were highly prevalent among this asymptomatic population. The benefit of introducing such STI screening programmes to improve reproductive health and HIV prevention efforts requires further study.

\section{Social and behavioural aspects of prevention oral session 1-Changes over time: evolution of individual and population level patterns 02-S1.01 DEVELOPMENTAL CHANGES IN MASCULINITY, SEXUAL
BEHAVIOUR, AND STI RISK AMONG ADOLESCENT BOYS}

doi:10.1136/sextrans-2011-050109.67

${ }^{1} \mathrm{M}$ Ott, ${ }^{2} \mathrm{D}$ Bell, ${ }^{1} \mathrm{~J} . \mathrm{D}$ Fortenberry. ${ }^{1}$ Indiana University School of Medicine, Indianapolis, USA; ${ }^{2}$ Columbia University Medical Center, New York, USA

Background Successful STI prevention for younger boys should tap into a cultural understanding of boys' romantic and sexual relationships. However, little data exist. We describe developmental changes in boys' relationships, masculinity, sexual behaviour, and STI risk across adolescence.

Methods After IRB approval, thirty-three 14-16-year-old boys were recruited from a teen clinic serving high STI prevalence urban areas, and participated in 3 one-hour qualitative interviews, approximately 6-9 months apart. Urine was tested for gonorrhoea, chlamydia and trichomonas using DNA-based tests. Over $80 \%$ were retained at each follow-up. Baseline interviews were coded, then each boy's three interviews were read as a group, looking for changes across the interviews in relationship experience, masculine beliefs, sexual communication and decision-making, sexual experiences, and STI prevention.

Results Mean age was 14.9 years, all were Medicaid-eligible (low income), 90\% were African American, and 16/33 were sexually experienced. We observed changes over time in relationships, masculinity, and sexual decision-making. At baseline, boys described girls as having more relationship power, with girls frequently initiating relationships and the decision to have sex. As boys gained experience, they perceived themselves as having more agency, and initiating sex more often. At baseline, we observed low levels of masculine beliefs, with many boys wanting meaningful and emotionally involved relationships, few viewing sex as a conquest, and most worrying about their own relationship and sexual competency. Three trajectories of masculinity emerge. A small number of Players" embodied a subset of masculine beliefs that included sex as a conquest, women as objects, and lack of emotional involvement. "Emotionally distant" was more common; these boys described initially trusting girls, "being burned" by someone they had been close to, and then not investing emotionally so as not to be hurt again. "Caretakers," also a small minority, emphasised men's role to protect and care for women. Sexual communication, mostly indirect or nonverbal at baseline, became more direct. Despite a baseline interest in sex within relationships, experiences of 1st sex were generally outside of relationships. 